\title{
$\beta$ Oscillations Are Linked to the Initiation of Sensory-Cued Movement Sequences and the Internal Guidance of Regular Tapping in the Monkey
}

\author{
Ramón Bartolo and $\oplus^{-H u g o ~ M e r c h a n t ~}$ \\ Instituto de Neurobiología, Universidad Nacional Autónoma de México, Querétaro, Querétaro. 76230, México
}

\begin{abstract}
$\beta$ oscillations in the basal ganglia have been associated with interval timing. We recorded the putaminal local field potentials (LFPs) from monkeys performing a synchronization-continuation task (SCT) and a serial reaction-time task (RTT), where the animals produced regularly and irregularly paced tapping sequences, respectively. We compared the activation profile of $\beta$ oscillations between tasks and found transient bursts of $\beta$ activity in both the RTT and SCT. During the RTT, $\beta$ power was higher at the beginning of the task, especially when LFPs were aligned to the stimuli. During the SCT, $\beta$ was higher during the internally driven continuation phase, especially for tap-aligned LFPs. Interestingly, a set of LFPs showed an initial burst of $\beta$ at the beginning of the SCT, similar to the RTT, followed by a decrease in $\beta$ oscillations during the synchronization phase, to finally rebound during the continuation phase. The rebound during the continuation phase of the SCT suggests that the corticostriatal circuit is involved in the control of internally driven motor sequences. In turn, the transient bursts of $\beta$ activity at the beginning of both tasks suggest that the basal ganglia produce a general initiation signal that engages the motor system in different sequential behaviors.
\end{abstract}

Key words: $\beta$ band oscillations; interval timing; putamen; rhesus monkeys; sequential behavior

\section{Introduction}

It has been suggested that $\beta$ activity $(12-30 \mathrm{~Hz})$ in motor areas is related to both the maintenance of the current state of the network (i.e., maintaining motion or holding), as well as the expectancy of forthcoming events (Engel and Fries, 2010). In addition, different studies support the notion that $\beta$ activity serves as a general coupling mechanism of neuronal activity within (Howe et al., 2011) and across brain structures (Kay and Beshel, 2010; Fujioka et al., 2012; Leventhal et al., 2012). The predictive role of $\beta$ activity in the motor system is evident during rhythm perception, where participants must extract regularities from sensory input and predict when the next beat will occur. For example, when human participants are instructed to extract the beat from a rhythmic pattern of ambiguous metrics, a peak of $\beta$ activity was observed just before the stimulus corresponding to the perceived downbeat (Iversen et al., 2009). Furthermore, enhanced $\beta$ activity was observed in the auditory cortex of participants passively listening to isochronous sequences of auditory stimuli, but not

\footnotetext{
Received Nov. 5, 2014; revised Feb. 11, 2015; accepted Feb. 12, 2015.

Author contributions: R.B. and H.M. designed research; R.B. and H.M. performed research; R.B. contributed unpublished reagents/analytic tools; R.B. and H.M. analyzed data; R.B. and H.M. wrote the paper.

This work was supported by CONACYT: 236836, PAPIIT: IN201214-26 grants to H.M. and CONACYT scholarship 202421 to R.B. We thank Sonja Kotz and Sundeep Teki for their fruitful comments on this manuscript. We also thank Luis Prado and Raúl Paulín for their technical assistance.

The authors declare no competing financial interests.

Correspondence should be addressed to Dr. Hugo Merchant, Instituto de Neurobiología, Universidad Nacional Autónoma de México, №. 3001 Boulevard Juriquilla, Querétaro, Querétaro 76230, México. E-mail: hugomerchant@unam.mx.

DOI:10.1523/JNEUROSCI.4570-14.2015

Copyright $\odot 2015$ the authors $\quad 0270-6474 / 15 / 354635-06 \$ 15.00 / 0$
}

when sequences were irregular (Fujioka et al., 2012). Interestingly, $\beta$ activity also occurred in motor areas, including the supplementary motor area (SMA) and the cerebellum during passive listening to isochronous stimuli, with an opposite phase compared to auditory cortical areas. These findings suggest that $\beta$ oscillatory activity provides a top-down motor expectation signal of the beat structure to the auditory system (Fujioka et al., 2012).

In a previous study, we reported modulations in putaminal $\beta$ activity while macaques performed isochronous tapping (Bartolo et al., 2014). In this task, called the synchronization-continuation task (SCT), macaques tapped first in synchrony with a sensory metronome for three intervals and then continued tapping at the same pace without sensory guidance for three more intervals, timing their behavior internally. We found that $\beta$ activity was mainly present during the internally driven phase of the task. Moreover, $\beta$ activity spread across large portions of the striatum and was highly coherent between distant recording sites. These results suggest that $\beta$ bursts reflect stable and sustained activity of a broad network involved in top-down control of internally driven isochronous tapping (Bartolo et al., 2014). Nevertheless, an unanswered question is whether striatal $\beta$ oscillations also play a role in the generation of nonperiodic movements (Teki, 2014). Although it is reasonable to expect a reduction in $\beta$ activity when stimulus occurrence is unpredictable, as observed in the human audiomotor system (Fujioka et al., 2012), it is important to consider that sensory cues elicit $\beta$ activity when stimuli are used to drive behavior (Leventhal et al., 2012). Thus, in the present work, we compare the $\beta$ band activity in the putamen during the exe- 
A
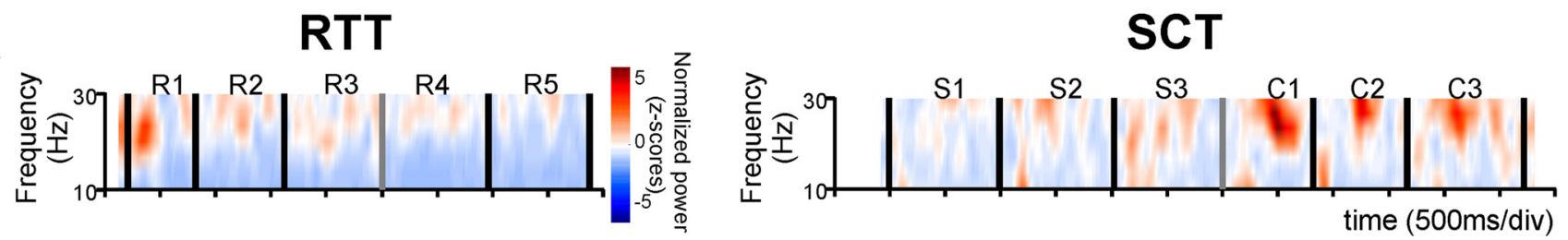

B
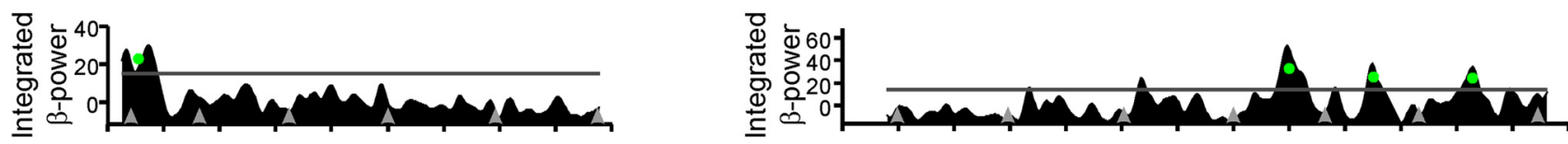

C
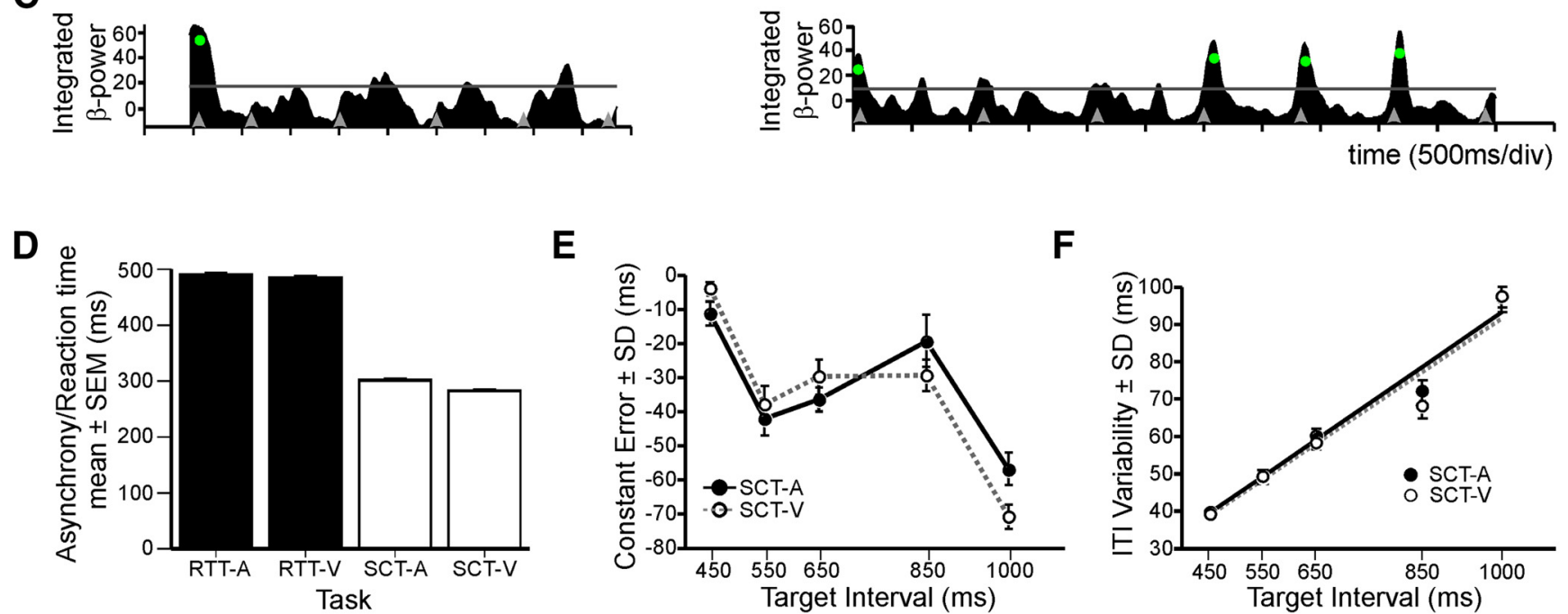

Figure 1. A, Normalized spectrogram of an example LFP recording tuned to serial order during irregular tapping (RTT) and isochronous tapping (SCT). In each plot, the time during task execution and spectral frequency components are represented in the horizontal and vertical axes, respectively. Black bars indicate the time of button taps, and on top of each plot the label of the respective serial-order element is shown. For the SCT, the interstimuli target interval was $1000 \mathrm{~ms}$. S1-S3 correspond to the sensory-guided phase of the task, whereas $\mathrm{C} 1-\mathrm{C} 3$ correspond to internally driven tapping. The normalized spectral power is color coded as indicated by the color bar. $\boldsymbol{B}$, Integrated $\beta$-power curves of the same LFP shown in $\boldsymbol{A}$. Gray horizontal lines show the threshold (mean +1 SD), and green dots mark valid modulations. Gray arrowheads mark the tap times. C, Power curves of a different LFP recording site. For the SCT, the interstimuli target interval was $1000 \mathrm{~ms}$ (same conventions as for B). D, Mean reaction times and asynchronies for the RTT and SCT are as follows: RTT [mean (SD)], audio (A), 489.6 (6.9); visual (V), 484.1 (5.6); SCT, A, 301.1 (13.8); V, 282.2 (13.7). $\boldsymbol{E}$, Constant error (mean ITI minus the target interval) of the monkeys' produced intervals. $\boldsymbol{F}$, Variability (SD) of the ITI as a function of target interval during the SCT. Solid and dotted lines are linear regressions of the data from the auditory $\left(R^{2}=0.97, p=0.002\right)$ and visual $\left(R^{2}=0.94, p=0.006\right)$ conditions, respectively.

cution of the SCT and a serial reaction-time task (RTT), in which monkeys performed a sequence of taps guided by aperiodic stimuli without the internally driven phase. Most of the recorded local field potentials (LFPs) had $\beta$ activity bursts associated with the beginning of a sequence of movements, independently of the task. After this initial signal, $\beta$ activity rebounded during execution of internally driven isochronous movements, emphasizing its relationship to predictive behavior and the internal set for processing regular events.

\section{Materials and Methods}

\section{General}

Two male monkeys (Macaca mulatta, 5-7 kg body weight) were trained to tap on a push button in two behavioral paradigms (see Tasks, below). Neurophysiological recordings were performed in the putamen during performance of the task (Bartolo et al., 2014). All of the animal experimental procedures were approved by the National University of Mexico Institutional Animal Care and Use Committee and conformed to the principles outlined in the Guide for the Care and Use of Laboratory Animals (NIH, publication Number 85-23, revised 1985).
Tasks

Serial reaction-time task. Monkeys were required to tap on a push button each time a stimulus was presented. Stimuli were separated by varying intervals randomly chosen between 600 and $1600 \mathrm{~ms}$. Each trial consisted of a series of six stimulus-response pairs, resulting in a sequence of five intertap intervals (ITIs; R1-R5; Fig. 1A, left). The intertrial period varied from 2 to $4 \mathrm{~s}$. Trials with reaction times between 200 and $500 \mathrm{~ms}$ were considered correct, and the animals received juice as reward. Stimuli were brief auditory tones $(33 \mathrm{~ms}, 400 \mathrm{~Hz}, 65 \mathrm{~dB})$ or visual stimuli $(33 \mathrm{~ms}$, $10 \times 10 \mathrm{~cm}$ square) in separate blocks. For each modality, 10 trials were collected in each recording site.

Synchronization-continuation task. This task has been described previously (Zarco et al., 2009; Merchant et al., 2011). Briefly, monkeys were required to tap on a push button, first guided by sensory isochronous stimuli (synchronization phase), to produce three intertap intervals (S1S3; Fig. $1 A$, right) and then to continue tapping without the sensory stimulus (continuation phase) to produce three more intervals (C1-C3; Fig. $1 A$, right). Five different interstimulus durations were used as targets in random order within a block. Trials with intertap intervals within a 30\% window from the interstimulus interval were considered 
correct. Auditory or visual stimuli were used as interval delimiters in separate blocks. Five correct trials of each target interval/modality combination were recorded in each recording site, for a total of 25 trials per block. Additional details have been reported previously (Bartolo et al., 2014).

\section{Neural recordings}

LFPs and single-unit activity were recorded in the putamen using a system with seven independently movable microelectrodes (1-3 M $\Omega$; Thomas Recording; Merchant et al., 2001). Electrodes were placed using guiding cannulas. Recordings were made at sites where single-unit activity was found. Recording sites changed from session to session. At each site, raw extracellular electric activity was sampled at $40 \mathrm{kHz}$. LFPs were acquired by low-pass filtering the raw signal (Thomas Recording; cutoff, $140 \mathrm{~Hz}$ ) and then downsampling the filtered signal at $571 \mathrm{~Hz}$. Recording sites were located using a structural MRI-based navigation system as reported previously (Bartolo et al., 2014).

\section{Data analysis}

General. Matlab routines (version 7.10.0.499, The MathWorks) were used for all of the analyses. LFP signals were aligned to each tap/stimulus in the task to look for associated power modulations. Intertrial data were discarded. Power modulations were quantified and further analyzed. Tap times and stimulus times were used to calculate stimulus-tap asynchronies, ITI duration, and variability.

Spectral analysis. The signal was aligned to stimulus or tap times to determine its association to sensory or motor events. Interevent (tap or stimulus) intervals could vary from trial to trial; hence, we constructed trimmed LFP time series for each trial before further analyses. Raw LFP time series were trimmed to the shortest interevent duration of all trials. Spectral quantities were estimated using the multitaper method (Thomson, 1982) implemented in Chronux 2.00 (Mitra and Bokil, 2008). The bandwidth for smoothing was always $10 \mathrm{~Hz}$ using one Slepian taper. Spectrograms were constructed trial by trial using a sliding window ( 300 ms width, $10 \mathrm{~ms}$ step) covering the whole LFP time series. Spectral density estimates were expressed as $z$ scores with respect to the mean and SD across all trials over the entire recording sessions. Time-varying modulations of spectral estimates were assessed on curves of integrated (trapezoid rule) $z$-scored power within the $\beta$ band $(10-30 \mathrm{~Hz})$ trial by trial. Valid modulations were defined as changes in the integrated power that were above a threshold (session mean $+1 \mathrm{SD}$ ) for at least $50 \mathrm{~ms}$ in at least three of the five trials per condition. Trial-averaged spectrograms were constructed for display purposes only.

Tuning analysis. Gaussian functions were fitted to the integrated power to determine tuning to serial order only for recordings with a significant effect revealed by a one-way ANOVA. The magnitude of valid modulations (mean power above threshold) for each intertap interval during the RTT was treated as the dependent variable in a nonlinear regression. Mean below-threshold integrated power was used when no valid modulations occurred. Serial order was the independent variable, according to the following equation:

$$
f(s)=h e^{-\left(\frac{\left\|s, s_{p}\right\|}{k}\right)^{2}}
$$

where $f(s)$ corresponds to the integrated power associated to a particular value of the independent variable $s, h$ is the parameter of maximum height, and $\kappa$ is the parameter of dispersion. $\left\|s, s_{p}\right\|$ is the Euclidean distance between $s$ and $s_{p}$, where $s_{p}$ corresponds to the preferred serial order. The function was fitted using the least squares method following a genetic algorithm (Kuri-Morales, 2004). A detailed analysis of the residuals was performed (Draper and Smith, 1981) and the $R^{2}$ value calculated. The statistical significance of the fittings was assessed using the corresponding ANOVA for the regression $(p<0.05)$. The peak of the fitted Gaussian was used to determine the preferred serial order, and distributions of this parameter were constructed for different subsets of recordings. Statistical comparisons were performed by obtaining cumulative distributions and applying $\chi^{2}$ tests. Hartigan's test was used to assess bimodality in the distributions (Hartigan and Hartigan, 1985).
Table 1. Number of LFP recordings with significant serial-order effects revealed by an ANOVA during the execution of the RTT and the SCT

\begin{tabular}{lcc}
\hline & Auditory & Visual \\
\hline Total analyzed & 285 & 285 \\
Sig. ANOVA SCT & 168 & 181 \\
Sig. ANOVA RTT & 238 & 211 \\
No response (no valid modulations) & 53 & 50 \\
Response SCT— beginning only & 10 & 8 \\
Response SCT—- continuation only & 81 & 62 \\
Response SCT— beginning and continuation & 141 & 165 \\
\hline
\end{tabular}

Bottom rows show LFP recordings classified by their response pattern during the SCT. Sig., Significant.

Table 2. Number (percentage) of LFP recordings with significant serial-order tuning during the execution of the RTT, the SCT, and both tasks, obtained using different LFP alignments (tap- vs stimulus-aligned data)

\begin{tabular}{lll}
\hline & Tap & Stimulus \\
\hline RTT only (Group 1) & $163(39.66)$ & $156(38.81)$ \\
SCT only (Group 2) & $144(35.04)$ & $118(29.35)$ \\
Tuned both tasks (Group 3) & $104(25.30)$ & $128(31.84)$ \\
\hline
\end{tabular}

Pooled data from the auditory and visual modalities were used to calculate proportions.

\section{Results}

\section{Spectral modulations in the $\beta$ band during the RTT}

Two monkeys performed a serial RTT, tapping on a push button in reaction to a sequence of six stimuli with random interstimulus interval (between 600 and $1600 \mathrm{~m}$ ). Visual or auditory stimuli were used in separate blocks of trials. Under these conditions, the animals performed irregular tapping movements using a reactive instead of a predictive strategy to drive their motor behavior (Zarco et al., 2009). The reaction times of monkeys were similar across modalities (Fig. 1D).

Using the multitaper Fourier transform (see Materials and Methods), spectrograms were obtained for the LFP signal recorded during the execution of the RTT and SCT (Fig. 1). We found transient spectral-power modulations in the $\beta$ band $(10-30 \mathrm{~Hz})$ during the execution of the tasks. From a total of 285 recording sites, a one-way ANOVA identified significant serialorder effects on the integrated $\beta$ power in 238 and 211 recording locations for the auditory and visual stimulus conditions, respectively (Table 1). At most of the recording sites, the magnitude of $\beta$-power modulations during the RTT was larger for the first intervals of the sequence (Fig. 1A, B, left). Accordingly, Gaussian fittings revealed tuning across the serial-order structure of the RTT (Table 2). Interestingly, the RTT distribution of preferred serialorder elements was biased toward the beginning of the task (Fig. 2A, gray lines), independently of the alignment of the spectrograms to the tapping times or stimulus times $\left(\chi^{2}\right.$ test on cumulative distributions, auditory stimuli, $p=0.29$; visual stimuli, $p=0.51$ ).

\section{Comparing $\boldsymbol{\beta}$ during the SCT and RTT}

The monkeys also performed the SCT, where they timed their taps guided by regular sensory stimulus during the synchronization phase followed by internally driven tapping during the continuation phase. Stimulus-tap asynchronies during the SCT were shorter than reaction times during the RTT (Fig. 1D). In addition, ITIs were slightly shorter than the target interval (Fig. $1 E$ ), and their variability increased as a function of the target interval $(F)$.

We also found recording sites that showed sequence-tuned $\beta$ activity during the execution of the SCT. Thus, the LFPs with sequence-tuned $\beta$ oscillations were divided as follows: only tuned during the RTT, only tuned during the SCT, and tuned in both tasks. Consistent with our previous report (Bartolo et al., 2014), 
the spectral power of $\beta$ activity during the SCT was larger during the phase in which regular tapping was internally driven rather than stimulus driven, as shown in the example spectrogram of Figure $1 \mathrm{~A}$ (right), particularly when the LFPs were aligned to the tap events. This bias toward the first two intervals of the continuation phase of the SCT was similar for the recording sites that were tuned only during the SCT or tuned in both tasks (Fig. 2A, top, black lines). On the other hand, the $\beta$ oscillations were larger only toward the beginning of RTT trials for LFPs tuned only during the RTT or in both tasks. Statistically significant differences in the preferred serial-order distributions were found between the SCT and RTT, regardless of whether they were tuned only to one or to both tasks (aligned to taps, $\chi^{2}$ test, $p<0.0001$; aligned to stimulus times, $\chi^{2}$ test, $p<0.0001$ ).

These findings suggest that striatal circuits are selectively engaged during internally driven timing behavior. However, the preferred serial-order distributions during the SCT on spectrograms aligned to the stimuli also revealed an additional $\beta$-oscillation peak at the beginning of each trial (Fig. $1 C$ ), resulting in a bimodal distribution (Hartigan's test, $p<0.001$; Fig. $2 A$, right). This initial $\beta$ peak was not obvious in distributions of tap-aligned spectrograms (Fig. 2A, left), revealing a stronger association with stimuli. Hence, these data suggest that, in addition to the $\beta$ activity during the internally driven phase of the SCT, there is a start $\beta$ signal in some LFPs that is associated with the initiation of the SCT sequence, similar to what was found in the RTT. Therefore, we analyzed the raw counts of valid $\beta$ modulations as a function of serial order for both RTT and SCT, and a large bias toward the beginning of the trial emerged in both tasks, particularly when aligned to the stimuli (Fig. $2 B$, right). The distributions of valid modulations not only resembled the distributions of preferred serialorder elements for both tasks when aligned to taps, but also reinforced the notion that $\beta$ activity bursts, when aligned to the stimuli, can signal the beginning of a sequential motor behavior in different temporal contexts (Fig. 2B, right).

\section{Discussion}

We found that striatal circuits exhibited $\beta$ activity bursts in the LFPs while monkeys performed both an irregularly (RTT) and a regularly (SCT) paced sequential tapping task. Bursts were tuned to the sequential structure of the task, decreasing their spectral power around a preferred serial order. The preferred serial-order distribution was strongly biased toward the beginning of the task during the RTT. In addition, a sequenceinitiation $\beta$ signal was observed during the SCT when LFPs were aligned to the stimulus times. This initiation signal was

A
Tap

Figure 2. $\quad \boldsymbol{A}$, Distribution of preferred serial-order for recording sites showing tuning across tasks and alignments. Top, Distribution of preferred serial-order for sites showing tuning during both the RTT and SCT. Preferred serial-orders obtained from recordings during the RTT (gray line) and the SCT (black line) are plotted separately. Bottom, Preferred serial-order for sites showing tuning only during one task (RTT or SCT). Left and right panels show distributions obtained from spectrograms aligned to tasks and alignments. Counts were normalized by the maximum number of valid modulations (SCT tap, 2072; SCT stimulus, 2221; RTT tap, 1195; RTT stimulus, 929). Intervals between SCT taps are labeled S1-S3 for the synchronization phase and C1-C3 for continuation. Intervals between RTT taps are labeled R1-R5.

followed by enhanced $\beta$ activity during the internally paced phase of the SCT. The generalized $\beta$-signal encoding the initiation of a regular or irregular tapping sequence, as well as the task specific $\beta$ increase during internally driven production of isochronous movements, was observed in sets of putaminal circuits that responded selectively during only one or during the two tasks.

There is growing evidence of the participation of the striatum in interval timing (Merchant et al., 2013a, 2015), and $\beta$ activity has been associated with the internal quantification of time (Fujioka et al., 2009, 2012; Iversen et al., 2009; Bartolo et al., 2014). Striatal $\beta$ activity has been associated with top-down control of behavior (Courtemanche et al., 2003), as in the execution of previously entrained responses (Howe et al., 2011) or the utilization of a sensory cue to guide behavior (Leventhal et al., 2012). In addition, $\beta$ activity is involved in the maintenance of a motor state during the internally driven production of regularly paced 
movements in the SCT (Bartolo et al., 2014), in which novel sensory influences should be overridden. Also, an increase in BOLD signal in the putamen has been associated with beat prediction in humans (Grahn and Rowe, 2013).

The findings of the present paper suggest that striatal $\beta$ activity also plays a role in cueing the initiation of a motor sequence. A general start signal mediated by $\beta$ activity was present during isochronous tapping as well as during irregular tapping, particularly when spectrograms were aligned to stimulus times. This pattern is consistent with the idea that $\beta$ activity reflects the utilization of a cue to initiate or cancel an action (Leventhal et al., 2012).

Thus, the observed initial and stimulus-driven $\beta$ bursts may reset the entire motor corticobasal ganglia-thalamocortical (mCBGT) circuit to engage in different sequential behaviors. Indeed, previous studies suggest that cell activity in the basal ganglia encode the first and last elements of behavioral sequences, allowing the concatenation of single movements into integrated sequential actions (Jin and Costa, 2010). Consequently, sequence bounding or triggering signals may reflect a general brain mechanism for packing individual behavioral elements as an entity (Fujii and Graybiel, 2003).

Previous studies (Yee et al., 1994; Teki et al., 2011; Merchant et al., 2013b, 2015) support the notion that the timing mechanisms are different when the intervals in a sequence are timed relative to a steady beat (beat-based timing) or not (absolute, or durationbased, timing). In beat-based timing, intervals are measured relative to a regular perceived beat, to which individuals are able to entrain, like in the SCT. In absolute timing, the duration of individual intervals is encoded discretely, like a stopwatch, and no entrainment is possible. The mCBGT circuit, including the SMA and the putamen, participates in beat-based timing (Grahn and Brett, 2007; Schwartze et al., 2011; Teki et al., 2011; Kung et al., 2013). Therefore, our results strongly support the notion that the mCBGT circuit is part of the beat-based timing mechanism, with a global entrainment in the $\beta$ band during the production of internally timed movements. On the other hand, the cerebellum takes part in absolute timing (Grahn and Rowe, 2009; Grube et al., 2010; Teki et al., 2011) or in the processing of temporal prediction errors, which can occur during the RTT (Teki et al., 2012). However, the mCBGT circuit and the cerebellum are not isolated systems. The subthalamic nucleus of the MCBGT circuit projects to the cerebellum, and the dentate nucleus projects to the neostriatum (Hoshi et al., 2005; Bostan and Strick, 2010). Consequently, the putaminal $\beta$ activity at the beginning of sensorycued motor sequences may also drive the cerebellum for the selection of irregular tapping sequences. The recruitment of cerebellar and striatal networks for timing of irregular and regular movement sequences is consistent with previous models that emphasize a synergistic context-dependent relationship between the two core timing systems in the brain (Teki et al., 2012; Schwartze and Kotz, 2013; Allman et al., 2014).

We found three groups of recording sites with serial-order selective $\beta$ activity: RTT only SCT only, or in both tasks. This suggests that different, although overlapping, striatal ensembles are recruited at the beginning of each task, engaging in synchronous $\beta$ activity. The engagement of the first ensemble may result in passing control to cerebellum-based networks during irregular tapping, whereas the engagement of the last ensembles may synchronize the mCBGT circuit for controlling internally driven movements. In the latter case, synchronous $\beta$ activity also carries temporal information (Fujioka et al., 2012; Bartolo et al., 2014). It is remarkable that individual circuits show both, the stimulus- related initiation $\beta$ signal and the movement-related top-down control $\beta$; this double role of $\beta$ activity could be associated with network reconfiguration. Finally, we observed a large preference for the initial intervals of the continuation phase followed by a sharp decrease at end of the SCT, which could be related to the change in the state of the network required for sequence termination. As shown previously (Leventhal et al., 2012), the decrease in $\beta$ power during last interval of the task could be also involved with the expectation for reward delivery.

The difficulty to initiate or terminate movements in Parkinson's disease (PD) patients has been associated with an overstabilization of mCBGT circuits through pathologically high $\beta$ activity. A hypothesis that could explain these movement problems based on the present data is that PD patients lack the sharp $\beta$ burst at the beginning of sensory-driven sequences. Actually, the problems that PD patients show during the initiation and termination of key piano movement sequences have been linked previously with deficits in $\beta$ activity modulation (Herrojo Ruiz et al., 2014).

In summary, the finding of a general start signal mediated by striatal $\beta$ activity is consistent with the use of a cue to initiate a sequence of regular or irregularly paced movements that has been previously concatenated through learning as an individual sequence. The subsequent suppression of putaminal $\beta$ activity during the RTT might be due to the recruitment of the durationbased cerebellar timing mechanism. In contrast, the enhanced putaminal $\beta$ activity during the internally driven production of isochronously timed taps is indicative of the information flow across the mCBGT network necessary to maintain a stable motor state during beat-based timing.

\section{References}

Allman MJ, Teki S, Griffiths TD, Meck WH (2014) Properties of the internal clock: first- and second-order principles of subjective time. Annu Rev Psychol 65:743-771. CrossRef Medline

Bartolo R, Prado L, Merchant H (2014) Information processing in the primate basal ganglia during sensory-guided and internally driven rhythmic tapping. J Neurosci 34:3910-3923. CrossRef Medline

Bostan AC, Strick PL (2010) The cerebellum and basal ganglia are interconnected. Neuropsychol Rev 20:261-270. CrossRef Medline

Courtemanche R, Fujii N, Graybiel AM (2003) Synchronous, focally modulated beta-band oscillations characterize local field potential activity in the striatum of awake behaving monkeys. J Neurosci 23:11741-11752. Medline

Draper NR, Smith H (1981) Applied regression analysis. New York: Wiley.

Engel AK, Fries P (2010) Beta-band oscillations-signalling the status quo? Curr Opin Neurobiol 20:156-165. CrossRef Medline

Fujii N, Graybiel AM (2003) Representation of action sequence boundaries by macaque prefrontal cortical neurons. Science 301:1246-1249. CrossRef

Fujioka T, Trainor LJ, Large EW, Ross B (2009) Beta and gamma rhythms in human auditory cortex during musical beat processing. Ann N Y Acad Sci 1169:89-92. CrossRef Medline

Fujioka T, Trainor LJ, Large EW, Ross B (2012) Internalized timing of isochronous sounds is represented in neuromagnetic beta oscillations. J Neurosci 32:1791-1802. CrossRef Medline

Grahn JA, Brett M (2007) Rhythm and beat perception in motor areas of the brain. J Cogn Neurosci 19:893-906. CrossRef Medline

Grahn JA, Rowe JB (2009) Feeling the beat: premotor and striatal interactions in musicians and nonmusicians during beat perception. J Neurosci 29:7540-7548. CrossRef Medline

Grahn JA, Rowe JB (2013) Finding and feeling the musical beat: striatal dissociations between detection and prediction of regularity. Cereb Cortex 23:913-921. CrossRef Medline

Grube M, Cooper FE, Chinnery PF, Griffiths TD (2010) Dissociation of duration-based and beat-based auditory timing in cerebellar degeneration. Proc Natl Acad Sci U S A 107:11597-11601. CrossRef Medline

Hartigan JA, Hartigan PM (1985) The dip test of unimodality. Ann Stat 13:70-84. CrossRef 
Herrojo Ruiz M, Rusconi M, Brücke C, Haynes JD, Schönecker T, Kühn AA (2014) Encoding of sequence boundaries in the subthalamic nucleus of patients with Parkinson's disease. Brain 137:2715-2730. CrossRef Medline

Hoshi E, Tremblay L, Féger J, Carras PL, Strick PL (2005) The cerebellum communicates with the basal ganglia. Nat Neurosci 8:1491-1493. CrossRef Medline

Howe MW, Atallah HE, McCool A, Gibson DJ, Graybiel AM (2011) Habit learning is associated with major shifts in frequencies of oscillatory activity and synchronized spike firing in striatum. Proc Natl Acad Sci U S A 108:16801-16806. CrossRef Medline

Iversen JR, Repp BH, Patel AD (2009) Top-down control of rhythm perception modulates early auditory responses. Ann N Y Acad Sci 1169:58-73. CrossRef Medline

Jin X, Costa RM (2010) Start/stop signals emerge in nigrostriatal circuits during sequence learning. Nature 466:457-462. CrossRef Medline

Kay LM, Beshel J (2010) A beta oscillation network in the rat olfactory system during a 2-alternative choice odor discrimination task. J Neurophysiol 104:829-839. CrossRef Medline

Kung SJ, Chen JL, Zatorre RJ, Penhune VB (2013) Interacting cortical and basal ganglia networks underlying finding and tapping to the musical beat. J Cogn Neurosci 25:401-420. CrossRef Medline

Kuri-Morales A (2004) Automatic clustering with self-organizing maps and genetic algorithms II: an improved approach. WSEAS Trans Syst 2:551-556.

Leventhal DK, Gage GJ, Schmidt R, Pettibone JR, Case AC, Berke JD (2012) Basal ganglia beta oscillations accompany cue utilization. Neuron 73:523536. CrossRef Medline

Merchant H, Battaglia-Mayer A, Georgopoulos AP (2001) Effects of optic flow in motor cortex and area 7a. J Neurophysiol 86:1937-1954. Medline

Merchant H, Zarco W, Pérez O, Prado L, Bartolo R (2011) Measuring time with different neural chronometers during a synchronization-continuation task. Proc Natl Acad Sci U S A 108:19784-19789. CrossRef Medline
Merchant H, Harrington DL, Meck WH (2013a) Neural basis of the perception and estimation of time. Annu Rev Neurosci:313-336.

Merchant H, Pérez O, Zarco W, Gámez J (2013b) Interval tuning in the primate medial premotor cortex as a general timing mechanism. J Neurosci 33:9082-9096. CrossRef Medline

Merchant H, Grahn J, Trainor LJ, Rohrmeier M, Fitch T (2015) Finding the beat, a neuro-computational approach. Philos Trans R Soc Lond Ser B Biol Sci 370:20140093. CrossRef Medline

Mitra PP, Bokil H (2008) Observed brain dynamics. New York: Oxford UP.

Schwartze M, Kotz SA (2013) A dual-pathway neural architecture for specific temporal prediction. Neurosci Biobehav Rev 37:2587-2596. CrossRef Medline

Schwartze M, Keller PE, Patel AD, Kotz SA (2011) The impact of basal ganglia lesions on sensorimotor synchronization, spontaneous motor tempo, and the detection of tempo changes. Behav Brain Res 216:685-691. CrossRef Medline

Teki S (2014) Beta drives brain beats. Front Syst Neurosci 8:155. CrossRef Medline

Teki S, Grube M, Kumar S, Griffiths TD (2011) Distinct neural substrates of duration-based and beat-based auditory timing. J Neurosci 31:38053812. CrossRef Medline

Teki S, Grube M, Griffiths TD (2012) A unified model of time perception accounts for duration-based and beat-based timing mechanisms. Front Integr Neurosci 5:90. Medline

Thomson DJ (1982) Spectrum estimation and harmonic analysis. Proc IEEE 70:1055-1096. CrossRef

Yee W, Holleran S, Jones MR (1994) Sensitivity to event timing in regular and irregular sequences: influences of musical skill. Percept Psychophys 56:461-471. CrossRef Medline

Zarco W, Merchant H, Prado L, Mendez JC (2009) Subsecond timing in primates: comparison of interval production between human subjects and rhesus monkeys. J Neurophysiol 102:3191-3202. CrossRef Medline 